\title{
Arbor
}

\section{El «Programa Poincaré» o funciones del matemático}

\author{
Javier de Lorenzo Martínez
}

Arbor CLXXVIII, 704 (Agosto 2004), 645-667 pp.

\section{Introducción}

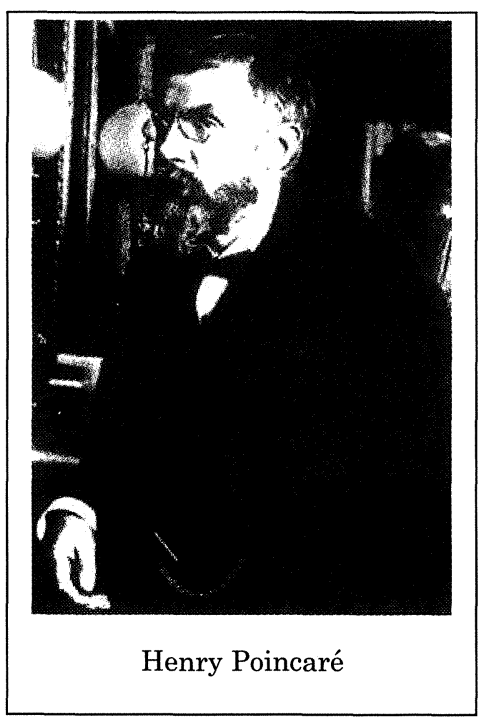

Ante todo, agradecer a la Real Academia de Ciencias Exactas, Físicas y Naturales estar hoy, aquí, ante ustedes. No ya a la Real Academia sino a las personas que lo han hecho factible: a José Manuel Aroca y, muy en especial, a Manuel López Pellicer.

Nos encontramos conmemorando un aniversario. Algo que puede convertirse en rutina, en autojustificación para reuniones; lo que, en cierta manera, está ocurriendo en otros campos. Aunque entre en esa rutina, me permito una sugerencia: Justamente este verano se cumplirá el centenario del nacimiento del Axioma de elección. Fue en 1904, en el Tercer Congreso Internacional de Matemáticos, cuando Zermelo lo formuló de manera explícita por vez primera. Y todos recordamos las inmediatas discusiones y polémicas a las que dio paso. Estaba en juego el papel de las definiciones y de las demostraciones existenciales, no constructivas. Y el problema, si cabe admitir o no la definición de un objeto sin poder mostrar, construir o calcular ese objeto.

Es tema al que dedican su análisis, su crítica y rechazo, y entre otros, los matemáticos de la Escuela de París, los componentes de la denominada escuela semi-intuicionista francesa-Borel, Lebesgue, Baire-. Por- 
que ligado al papel de las definiciones no constructivas se encuentra como problema la existencia o no del infinito actual, de si cabe ir más allá del infinito numerable. Como sugerencia, se podría dedicar una Sesión a conmemorar el nacimiento del Axioma de elección así como sus repercusiones, su papel en el Hacer matemático...

\section{El programa: funciones del matemático}

Poincaré nace en Nancy el 29 de Abril de 1854. Muere en París el 17 de Julio de 1912. Considerado en su época como el matemático más brillante del momento, puede afirmarse hoy que ha sido uno de los mayores matemáticos y físicos creadores de todas las épocas. Como de alguno de sus temas matemáticos específicos van a tratar quienes me acompañan en este Sesión, voy a limitarme a esbozar un rápido panorama de lo que Poincaré consideraba la misión que todo matemático debe ejercer: las funciones o papeles que le corresponden como matemático y que él trató de cumplir con toda honestidad a lo largo de su vida, que trató de «vivir matemáticamente».

Funciones o papeles del matemático que constituyen, realmente, un Programa de cómo enfocar la Matemática, de cómo hacer matemática no ya en el momento en el que las enuncia, sino para el porvenir. Poincaré no expone problemas para resolver en el futuro sino que, simplemente, ejemplifica con temas amplios esas funciones que exige cumplan los matemáticos.

Es un Programa, que denomino Programa Poincaré, que explicita en el Primer Congreso Internacional de Matemáticos (CIM), celebrado en Zürich en Agosto de 1897. Y donde enuncia, a la vez, una convicción, muy profunda, con todas sus consecuencias, que son muchas: la Matemática es un hacer de alguien que la trabaja y produce, del matemático. Es este quien la construye en un proceso siempre inacabado. No hay, para Poincaré, un mundo eidético de objetos, de propiedades de unos objetos que el matemático tenga que descubrir; mundo eidético hecho no se sabe muy bien por quién ni para qué y al que tampoco se sabe muy bien cómo acceder. Para Poincaré, el producto matemático depende del matemático individual, pero este trabaja en sociedad, con unos temas y problemas ya dados, en relación, y a veces disputa, con otros matemáticos. Las diferentes disciplinas son construcciones que sacamos enteramente de nuestro espíritu. (VC 137)

Centrados en el «Programa Poincaré», las funciones o papeles que asigna a la Matemática, al matemático, son: 


\section{Estética}

Es el trabajo en sí del matemático. Plantear y resolver problemas, intuir analogías, demostrar proposiciones, crear nuevos campos de trabajo... Aportes de este fin estético o intrínseco al Hacer matemático, y como meros ejemplos, se le puede considerar el padre de la Topología -especialmente de la Homotopía y la Topología algebraica, donde formula la Conjetura de Poincaré precisamente hace ahora un siglo y donde ya ha establecido el contraejemplo que supone el dodecaedro de Poincaré-, de las funciones fuchsianas o automorfas, de las ecuaciones diferenciales cualitativas, de la geometrización de todas las ramas de la matemática y la física, de la importancia del Cálculo de Probabilidades para el razonamiento matemático, hasta de su anticipación a Teorías como la del caos determinista y la de fractales...

Con una advertencia: para Poincaré la Matemática no tiene como misión mirarse el ombligo (CM p. 141), no puede encerrarse en una torre de marfil. Ello supondría que a corto o largo plazo el matemático quedaría estéril, esterilidad velada por el aparente desarrollo de miles de teoremas, de simples combinaciones formales de palabras, de fórmulas carentes de importancia, sin contenido matemático alguno. Aunque esas combinaciones fueran correctas lógicamente y se mostrara que los teoremas se obtienen válidamente de los axiomas, ello no significaría nada porque

Reduciendo el pensamiento matemático a una forma hueca, se le mutila. (CM p. 115)

Por ello el matemático, la Matemática tiene otro papel, otra función, que es la

Física

Se trata de suministrar un instrumento para el estudio de la physis y se centra en el enlace de la Matemática con otros haceres, con otras disciplinas. Especialmente, estamos a primeros del s. XX, con la Astronomía, la Física.

En este campo hay que tener presente una división de papeles. Lo que aporta la Matemática a las demás ciencias, lo que la Matemática recibe de ellas.

La Matemática aporta a la Ciencia el único lenguaje que esta maneja. Si la ciencia es un lenguaje bien hecho, lo es porque dicho lenguaje es el matemático. Pero además la Matemática ayuda al cálculo, 
a la resolución de ecuaciones o de sistemas de ecuaciones sean diferenciales o algebraicas... Y si hasta aquí lo aportado es algo admitido generalmente, para Poincaré hay algo más: la Matemática aporta una ayuda para la invención del científico al desarrollar estructuras que pueden posteriormente interpretarse en distintos terrenos. En otras palabras, el matemático aporta la capacidad de ver analogías. Y la analogía se le muestra como el instrumento clave para la invención tanto matemática como científica. Con lo cual el Hacer matemático permite al Físico

hacerle conocer la armonía oculta de las cosas haciéndoselas ver desde un nuevo ángulo. (VC 147)

Aquí Poincaré va a enunciar otra de sus creencias básicas respecto al papel de la Lógica y la invención o creación matemática y científica. Se pregunta: ¿cómo tratar las ecuaciones de la física matemática? Para algunos se trata de obtener simplemente las consecuencias y considerar estas como realidades intangibles. Sin embargo para Poincaré el trabajo del científico no ha de limitarse a deducir sino que ha de buscar las analogías profundas, la armonía que se oculta en los fenómenos de la naturaleza. Y para ello las ecuaciones de la física deben enseñarnos

Lo que se puede y debe cambiar (VC 146)

El matemático, a su vez, recibe aportes de los terrenos científicos. Desde ellos se le plantean problemas que debe resolver y que le llevan a crear nuevos campos de investigación, a modificar incluso los conceptos que maneja. Así la serie de Fourier ha surgido de un problema físico pero se ha convertido en un instrumento precioso del Análisis y ha forzado a estudiar las funciones discontinuas, por ejemplo, ha forzado a cambiar la propia noción de función, lo que quizá no hubiera ocurrido desde el interior de la Matemática.

Pero hay algo más para Poincaré y es tema en el que quiero insistir porque ha sido uno de los campos que más ha dividido a la comunidad científica y ha vuelto a plantearse en el último tercio del siglo XX con radicalidad absoluta: la separación y el abismo producidos entre el matemático "puro» y el físico "puro», abismo que ha atravesado la Matemática y la Física a lo largo del s. XX. Para Poincaré, el matemático también recibe soluciones y demostraciones a los problemas por parte del físico. En sus palabras: 


\section{El «Programa Poincaré» o funciones del matemático}

La física (...) nos ayuda a encontrar los medios, y eso de dos maneras: Nos hace presentir la solución; nos sugiere los razonamientos. (VC. 152)

Con un matiz, las demostraciones que sugiere el físico quizá carecen del rigor que puede exigir el matemático, pero no por ello son menos demostraciones. Poincaré afirmará: «no seamos tan puristas» porque ese rigor informal que aporta el físico en las soluciones y demostraciones matemáticas es un tipo de rigor que, en el fondo, permite al matemático encontrar la vía para alcanzar la solución o elaborar la demostración matemáticas que, de otra manera, le sería más difícil o, incluso, imposible de hallar. Entrevisto el camino, asegurada la existencia de una solución, de una demostración, ya se tiene parte de la solución, ahora pretendidamente rigurosa o matemática, del problema, de la demostración.

No sólo sugiere las líneas demostrativas, sino que también la Física devuelve al matemático el mismo instrumento que este le aportaba: la analogía. Una analogía que ahora se apoya en la imagen no ya geométrica, sino física. Y las imágenes físicas posibilitan ver de un golpe lo que la deducción pura sólo mostraría sucesivamente. Gracias a estas imágenes el matemático acopla los elementos dispersos de la solución que le llevan a una clase de intuición por la cual adivina antes de poder demostrar.

Así, en el caso de las membranas vibrantes, la naturaleza física del problema muestra de antemano la existencia de una sucesión de valores propios y sólo después de captar esta «demostración» física, Poincaré fue capaz de demostrar la existencia matemática de los valores propios correspondientes.

Poincaré confiesa, realmente, su método de trabajo: es la analogía -a veces estructural, a veces geométrica, a veces estrictamente física- la que le permite presentir, intuir la solución que no está en condiciones de establecer por un razonamiento deductivo. Una forma de trabajar la matemática en la cual se manifiesta

Nuestra necesidad de pensar en imágenes (CM p. 102)

Forma de trabajar por la cual el mismo Hermite le calificará de

vidente a quien las verdades se le aparecen con una luz intensa, pero fundamentalmente a él solo. (Carta a Mittag-Leffler de 22 de Noviembre de 1888. Tomado de Gray, p. 32)

La convicción de Poincaré respecto a la unidad intrínseca de la Matemática y la Física constituye una posición que va en línea con algunas 
tendencias que han ido surgiendo en el último tercio del s. XX. Me basta recordar aquí las discusiones en torno a los trabajos de Donaldson y sus «demostraciones físicas» y no «estrictamente matemáticas», por ejemplo, donde las ideas físicas son las que sugieren y hacen presentir no sólo los problemas sino sus tipos de solución y razonamiento; o las exigencias de Jaffe, de Arnold, de Atiyah pidiendo un mayor enlace entre Física y Matemática intentando superar la barrera creada entre estos dos campos, una barrera que a Poincaré se le antojaría como auténtica pérdida para todos, matemáticos y físicos, porque los fines Estético y Físico se le muestran inseparables. En concreto, unidad en el Análisis puro y, en él, en las ecuaciones diferenciales, pero también la noción de grupo que materializa la simetría inherente a la physis, a nuestro conocimiento de la misma. Barrera que, en estos momentos, también habría que eliminar entre otros campos y no sólo con la Física como algunos actos del recordado Año Mundial de las Matemáticas han puesto de relieve.

En esta función, la de enlazar Matemática y Física, Poincaré trabajó en Mecánica Celeste con aportaciones ligadas a los sistemas dinámicos con sensibilidad a las condiciones iniciales; creó su Teoría de la relatividad restringida, se lanzó a esbozar las ecuaciones que podrían regir el extraño mundo de los quantos, de una Mecánica cuántica que estaba en sus albores y nadie sabía muy bien por dónde podría continuar...

\section{Filosófica}

Por lindar no sólo con la Física sino con la Filosofía, hay un tercer papel que ha de cumplir el matemático en su trabajo: el Filosófico. La Matemática, el matemático ha de ayudar a profundizar, a clarificar en lo posible conceptos como los de Espacio, Tiempo, Número. Pero también tiene que analizar críticamente su propia praxis, aquello que hace y cómo lo hace. No basta crear Matemática; no basta crear Ciencia. Para Poincaré el matemático tiene la obligación de pensar críticamente en lo que hace.

Es aquí donde, realmente, tendría que centrar el tema: ¿qué piensa Poincaré de lo que hace y de cómo lo hace? Responder a esta pregunta constituiría lo que calificar de exposición de la Filosofía de la Matemática de Poincaré, de su pensamiento matemático. Pero también de su Filosofía de la Ciencia porque ambas, para él y como he indicado, están íntimamente entrelazadas. Me limito a exponer, dado el tiempo asignado, alguno de los temas centrales de dicho Pensamiento, marginando aquí su Convencionalismo geométrico, su Convencionalismo científico que más 
bien debería ser calificado como Realismo estructural científico. (Para un estudio más comprensivo remito tanto a mi libro 1974 como al ensayo 'Poincaré, pensador de la matemática' a publicar por la Universidad Politécnica de Barcelona).

\section{Algunos temas del pensamiento filosófico matemático de Poincaré}

El número natural y la inducción completa

Si el matemático construye objetos, estructuras y busca y demuestra propiedades de las mismas no construye a partir de la nada. Como dato primario, fundamental, se le muestra el número natural. Afirmará reiteradamente

El único objeto natural del pensamiento matemático es el número natural (VC,149).

Los demás, como los números reales, los ha inventado a partir de los naturales. Pero si los ha inventado ha sido porque se ha visto forzado a inventarlos por influjo del mundo exterior. El número natural se apoya en la potencia del espíritu que se sabe capaz de reiterar una acción desde que esa acción es posible. Y se sabe capaz de esa posibilidad no por un razonamiento deductivo, lógico, sino por una captación intuitiva. Reiterar una acción como la de "sucesor», el "uno más», conduce a elaborar la sucesión de los números naturales. Para razonar sobre esta sucesión que posibilita captar el infinito potencial, nunca clausurado, el matemático maneja el razonamiento por inducción completa. Razonamiento que refleja esa misma potencia y constituye, para Poincaré

\section{El razonamiento matemático por excelencia $(\mathbf{C H}, 65)$}

Aparecen, así, el número natural, la noción sucesor y la inducción completa como manifestaciones de la potencia del espíritu humano, como los datos básicos en los que fundamentar o apoyar el Hacer matemático (Cfr. especialmente CH cap. I). La inducción completa constituye, para Poincaré, y adoptando la terminología kantiana, un juicio sintético a priori, realmente una precondición para la construcción matemática. Una terminología algo confundente porque el a priori en Poincaré no es trascendente sino un elemento constitutivo que el espíritu humano ha ido incorporando durante la evolución como especie. Incorporación efectuada en su lucha por la supervivencia aquí en la Tierra. En otros luga- 
res, en otros mundos como en uno gelatinoso, la especie que tratara de sobrevivir en ellos habría tenido que constituir otras precondiciones, otros a priori. En la Tierra hay cuerpos «sólidos», entre ellos el propio cuerpo humano, que reitera sus pasos al andar, y da un paso y después otro y así sucesivamente...

Por otro lado, la inducción completa permite sintetizar en único bloque una infinidad de silogismos. De ahí su generatividad apoyada en la posibilidad reiterativa. Y de ahí que sea irreducible a la Lógica porque esta se apoya en el principio de identidad y, consecuentemente, carece de la generatividad del principio matemático.

No es el único a priori como en general se le ha achacado. De modo explícito, en CM p. 116, afirma la existencia de otros juicios sintéticos a priori equivalentes al de inducción completa y que se apoyan o basan en la misma capacidad reiterativa por lo cual poseen idéntica capacidad generativa; también la noción de grupo es un a priori como reconoce de modo explícito, tema al que volveré más adelante.

Derivación lógica - Demostración matemática

Enlazado con lo anterior, Poincaré trata de precisar y diferenciar la auténtica demostración matemática de la demostración lógica, de lo que estima simple verificación. Si es por intuición como se inventa, es por lógica como se demuestra, será una de sus expresiones reiteradas. Pero hay que precisar, por supuesto. Lo que importa es el contenido matemático y éste sólo es captable por la intuición que es la verdadera fuente de la invención. Ahora bien, la intuición, a veces, engaña. De aquí la necesidad de una comprobación, de lo acertado de la misma. Este es el papel que aporta la demostración: comprobar que lo intuido es correcto.

Hay aquí dos planos, implícitos: por un lado, enfocar la demostración como proceso verificacional o constructivo; por otro, considerarla como objeto ya dado y entonces hay que caracterizarlo. Existencia de dos planos, matemático y meta-matemático en la cual insistirá, y ya de modo explícito, cuando realice su crítica a la ponencia de Hilbert ante el tercer CIM donde el matemático de Göttingen esboza un primer intento de fundamentación de la matemática; diferencia de planos que sólo en los años veinte Hilbert hará suya.

Desde el plano lógico una demostración es una sucesión de fórmulas tales que las primeras son axiomas y las restantes se obtienen de las anteriores mediante unas reglas de deducción previamente explicitadas; la última fórmula de la sucesión es el teorema que se pretendía demostrar. 
En esta derivación lo único que se exige es aplicar correctamente las reglas y se pide abandonar cualquier idea o contenido semántico. Con lo cual

Para demostrar un teorema no es necesario ni útil saber lo que se quiere decir. (CM p. 114).

Para Poincaré es una caracterización formal que no da cuenta, para nada, de lo que para el matemático es una demostración. Ésta parte precisamente del final, del teorema a demostrar, no de los axiomas, y el matemático va guiado por una idea aportada precisamente por la proposición planteada. Es la intuición la que guía el proceso demostrativo.

Ahora bien, una vez realizada resulta que la intuición también se presenta esencial para la derivación formal, a pesar de lo que indiquen los formalistas porque la clave se centra en aplicar las reglas correctamente. Y esa aplicación sólo es factible por la intuición de qué regla aplicar y cómo aplicarla en cada caso. No hay mecanicismo formalista o sintáctico alguno en el proceso demostrativo. Es la intuición la que permite ver por qué se eligen unas reglas en lugar de otras; la que permite captar las líneas centrales, el «alma» de la misma; la que permite ver por qué se eligen unos principios en lugar de otros...

Todo ello implica, una vez más, la nítida separación que existe entre Lógica y Matemática. Incluso cabe estimar que es la misma lógica la que cae bajo el hacer matemático porque la propia noción de derivación exige la reiteración: es una sucesión en la cual a una fórmula le sigue otra que es su sucesora...

En este sentido la axiomatización al estilo de la escuela italiana y que asume Hilbert como campo propio, hasta el punto de convertirse en el campeón de «el método axiomático» para definir implícitamente los objetos matemáticos, se le muestra a Poincaré como proceso que sólo encuentra su justificación si se demuestra que dicha definición axiomática es consistente, no contradictoria. De aquí que la primera exigencia de la pretendida axiomatización sea dicha demostración, que ha de ser directa y no por el dato de un modelo o realización de dicha definición axiomática como ocurre con las geometrías no-euclídeas. Algo que en el caso de la Aritmética y de la Teoría de conjuntos de Zermelo no se ha conseguido y Poincaré encuentra difícil que se pueda realizar dicha demostración sin utilizar la inducción completa que es, precisamente, uno de los principios que se admiten como axiomas en la definición implícita, en la axiomatización al estilo Peano y que asume Hilbert. 
El continuo y su discretización

El continuo es, como magnitud extensa, un elemento intuido, que se muestra incluso como un a priori respecto al punto y, por ello, no puede ser reducido a lo discreto. Un dato primario, al igual que el número natural, y donde lo geométrico queda subsumido por lo topológico. Sin embargo parece que los matemáticos han discretizado ese continuo y lo han convertido en un conjunto de puntos separados entre sí, el conjunto actualmente dado de números reales.

En un intento de justificar la construcción de los números reales, de esa discretización del continuo, Poincaré elabora unos estudios propios de lo que posteriormente se ha denominado Epistemología genética. Trata de aclarar cómo la especie humana, el matemático en ella, ha tenido que construir ese número real forzado por el mundo exterior en el sentido de que tiene que medir y pesar, no sólo contar. Medir la diagonal de un cuadrado, por ejemplo, fuerza a elaborar la noción de número inconmensurable, de número irracional. Y no sólo en el caso de la medida, porque también al pesar aparecen contradicciones que hay que superar. De manera modélica Poincaré muestra cómo poder superar la contradicción que se presenta al pesar tres cuerpos y encontrar que los pesos cumplen $\mathrm{A}=\mathrm{B}, \mathrm{B}=\mathrm{C}$, pero $\mathrm{A} \neq \mathrm{C}$. Y ello obliga a insertar pesos intermedios que sean más aproximados y reiterar -nuevamente la reiteración como apoyatura básica- la operación. Creados los números reales, como símbolos que den cuenta de ese intercalamiento y aproximación adecuados, hay que establecer las operaciones que deben cumplir manteniendo siempre las ya obtenidas en campos numéricos previos...

\section{El problema ontológico y el infinito actual}

La existencia de los números reales le conduce a Poincaré a indicar que son símbolos, elementos realmente ideales, con los que representar puntos y límites entre magnitudes así como representar el valor numérico asociado a esas magnitudes extensas, al continuo. Admitirlos como objetos reales conduciría a aceptar la existencia del infinito actual. En este punto no hay que olvidar una de las convicciones centrales de Poincaré a la que hice referencia: la Matemática es un producto de la especie humana en su interrelación con la naturaleza. Y el hombre es un ser finito. De modo consecuente el único infinito real que puede construir, que puede manejar es el infinito potencial y, en cuanto a la cardinalidad, el cardinal numerable. 


\section{El «Programa Poincaré» o funciones del matemático}

$\mathrm{Al}$ difundirse las paradojas cantorianas por influjo de Russell, Poincaré manifestará que se deben a que los cantorianos han olvidado que el único infinito que cabe aceptar es el potencial y han admitido, sin más precaución, el infinito actual como un existente real, no simbólico. A pesar de ello, estudiará el origen o causa de las paradojas. Causa que encuentra en la impredicatividad o Principio de Círculo Vicioso ligado, precisamente, a la admisión de ese infinito actual.

Ello no es obstáculo para que se hable del infinito pero siempre que se sepa que puede traducirse a términos finitos. Ello implica que los conjuntos han de ser dados por una ley predicativa, ley que permita obtener cada uno de los términos del conjunto sin hacer referencia al total de los mismos, obtención que ha de ser factible, calculable en un número finito de pasos. De aquí que no tiene sentido hacer referencia al total de los elementos de un conjunto que jamás podrán ser nombrados, jamás podrán ser calculados, como hacen cantorianos y pasígrafos -logicistas a lo Russell- que admiten la existencia de conjuntos no numerables. De modo consecuente las definiciones puramente existenciales se le muestran, por ello, rechazables salvo que, posteriormente, pueda calcularse, de modo efectivo, el objeto definido.

En este punto la caracterización conjuntista del número natural apoyada en la noción de conjunto, biyección entre conjuntos y clase de equivalencia de conjuntos por la cual los números naturales no son otra cosa que conjuntos finitos -más bien clases de equivalencia de conjuntos bi yectivos con un conjunto finito-, se le muestra totalmente circular -requiere de la previa noción de unidad y reiteración de la misma- además de artificial y con una apoyatura ontológica que considera errónea. No se capta intuitivamente la noción de conjunto -y mucho menos la de conjunto infinito- sino que ésta se construye mediante la ley correspondiente.

Los conceptos de Espacio y Tiempo. La Geometría, reflejo de la estructura de grupo

Respecto al Espacio y el Tiempo, para Poincaré no existen ni el Espacio absoluto ni el Tiempo absoluto. En cuanto al tiempo no hay simultaneidad de acontecimientos. Son dos conceptos que se ligan, estrechamente, a lo que considerar Física y, como tales, entran en juego en Teorías como la de la Mecánica clásica o newtoniana, la de la relatividad especial. Teoría en la cual y años antes de que Einstein eligiera el ejemplo de los relojes para dar cuenta de la no simultaneidad lo había hecho Poincaré con radical simplicidad expositiva. 
Pero son conceptos que no sólo se ligan a la Física, sino en el caso del Espacio a la Geometría. De modo clásico la Geometría se había considerado como la ciencia que estudia las propiedades del espacio. Sin embargo, escribe en 1895

La geometría no tiene por única razón de ser la descripción inmediata de los cuerpos que caen bajo nuestros sentidos, es ante todo el estudio analítico de un grupo.

En este punto Poincaré vuelve a realizar un estudio de carácter epistemológico genético para poder precisar el origen de las nociones y de los axiomas o principios geométricos -que estima como «definiciones disfrazadas» o convenciones de esas nociones geométricas- y de aquellas geometrías que ha manejado el matemático. En su lucha por la supervivencia aquí en la Tierra la especie humana ha elaborado la Geometría que más le ha convenido para dicha supervivencia: la Geometría métrica euclídea. Y lo ha hecho forzando la existencia constitutiva de otro juicio sintético a priori: la noción de grupo. En la naturaleza terrestre existen cuerpos que, en principio son rígidos, como un palo, la mesa en la que me apoyo, mi propio cuerpo. Esos cuerpos sufren transformaciones tanto de estado como de posición. En este último caso hay unas transformaciones básicas que mantienen invariante el estado del cuerpo: los desplazamientos. Los desplazamientos, su composición, forman la estructura de grupo. Noción de grupo que termina incardinándose como un juicio a priori en el individuo, lo mismo que ocurrió con la inducción completa y el proceso de reiteración de una acción desde que esa acción se hacía posible.

Las geometrías aparecen como el estudio de unos grupos determinados cuando se les agrega una $u$ otra métrica, una u otra noción de distancia, noción que es siempre convencional. La especie humana ha construido en primer lugar la Geometría métrica euclídea porque es la que le ha resultado más útil en el entorno local en que los individuos se mueven sobre la superficie terrestre. Una Geometría que ha dado paso a la creación conceptual de un Espacio homogéneo, isótropo, ilimitado e infinito. Un espacio que, por supuesto, es lo más antiperceptivo que se pueda imaginar. Poincaré ha tratado de precisar las diferencias entre lo que llamar espacio representativo y espacio conceptual, único en el cual podemos representar, por ejemplo, la línea recta.

Por su lado, la Geometría Proyectiva -la geometría del ojo frente a la geometría del músculo como la euclídea-, ha tenido que aguardar al siglo XIX para aparecer. Lo mismo las geometrías métricas no-euclídeas con sus espacios conceptuales asociados correspondientes. Si cualquiera 


\section{El «Programa Poincaré» o funciones del matemático}

de estas geometrías hubiera sido más conveniente para la supervivencia de la especie humana esta las habría llegado a construir antes que la mé trica euclídea.

Gracias a la métrica euclídea se ha podido construir una teoría como la Mecánica clásica. Si en el futuro el estudio de la naturaleza obligara a elegir otro tipo de geometría para captar esa physis, no habría problema para ello pero, de momento, la métrica euclídea nada tiene que temer y no lo tendrá en el ámbito local. Y no lo tiene tanto por su utilidad como por su simplicidad: el espacio al que da paso es de curvatura constante e igual a cero lo que facilita la expresión de sus fórmulas.

\section{Tipologías de matemáticos}

Hay muchos tipos de individuos $\mathrm{y}$, consecuentemente, de matemáticos. Poincaré establece dos tipologías según el modo de trabajar y según la ontología que los matemáticos profesan. En cuanto al modo de trabajar apunta la existencia de dos grandes bloques: los intuitivos o geómetras -entre los cuales incluye a Riemann y habría que hacerlo con él mismo- y los lógicos o analistas -cuyo modelo básico es Weiertrass-.

Pero según la ontología que los matemáticos profesan, se escinden en otros dos bloques: realistas que creen en la existencia de un mundo eidético de entidades matemáticas que hay que descubrir -y el modelo que presenta es su maestro Hermite-y pragmáticos que, como él, consideran que los objetos matemáticos son construcciones del matemático, construcciones de la razón humana que es un simple relámpago en medio de una noche oscura, pero sabiendo que ese relámpago lo es todo y que, por ello mismo, el mundo matemático no es un mundo cerrado, ya dado de una vez para siempre, sino que es un hacer en permanente devenir.

$\mathrm{Y}$, ya puestos, Poincaré trata de analizar la invención matemática; al menos conoce un caso, el suyo. Y lo expone con vívida descripción.

Conviene, aquí, una precisión: para Poincaré el matemático nace, no se hace. Lo cual no implica que la educación, la enseñanza no se le muestren absolutamente imprescindibles. Porque se nace en un contexto social y en una época determinada. Según ese contexto social, según la época, ese matemático en ciernes podrá llegar a ser matemático o no, se centrará en unos determinados temas y problemas y no en otros. 
¿Hay una fundamentación definitiva del Hacer matemático?

Hay filósofos y matemáticos que pretenden encontrar un fundamento seguro y cierto, ya definitivo, para la Matemática. Logicistas como Russell y Couturat, siguiendo a Frege, encuentran ese fundamento en la Lógica, aunque una Lógica transformada en logística basada en una ideografía al estilo pasigráfico de Peano. Los cantorianos, por su lado, creen fundamentar la matemática en la teoría de conjuntos que supone partir del infinito actual para, desde él, y mediante toda una compleja maquinaria de cardinalidades transfinitas, alcanzar lo finito. Los formalistas y hilbertianos se apoyan en el signo como la clave de todo el hacer matemático, signo acompañado de la derivación formal sintáctica a partir de unas posiciones previamente dadas, los axiomas, para ir obteniendo los teoremas matemáticos al estilo de la legendaria máquina de Chicago, en la cual se introduce el cerdo vivo por un lado y por el otro sale transformado en jamones y salchichas.

Para Poincaré, todos los que pretenden obtener ese fundamento ya definitivo del hacer matemático son profetas de desdichas. Y los profetas de desdichas, por fortuna, han fracasado siempre. No se pueden buscar unos fundamentos ya para siempre porque eso supondría que la Matemática se encontraría clausurada y podrían deducirse todos sus teoremas de sólo unos cuantos principios o axiomas como pretenden esos profetas, los fundamentalistas. Si se puede hablar de un fundamento este sería la razón humana y su interrelación con la physis. Es la razón la que construye la matemática y busca sus aplicaciones y, para esa razón, no hay un camino plenamente determinado y ya dado para siempre. Nuevos problemas provocarán nuevos desarrollos, incluso la posible aparición de nuevos juicios sintéticos a priori y no sólo de nuevas teorías... Es la razón la que, afortunadamente y hasta ahora, no se encuentra clausurada y, por ello, tampoco el Hacer matemático.

\section{Al servicio de la sociedad}

Si los tres papeles o funciones antes esbozados son los que Poincaré asigna de modo explícito al matemático, ya he indicado que este nace en sociedad. Y la Matemática ha surgido de una necesidad de la especie humana: la de sobrevivir sobre la Tierra. La Matemática, por ello, también está obligada a cumplir otro papel: La Matemática ha de estar al servicio de la Sociedad. Al servicio, pero con sus matices correspondientes. La Matemática, como la ciencia en general, debe ser cultivada no sólo por 
sus aplicaciones. Frente al convencionalismo nominalista representado en su época por Le Roy, Poincaré alzará la necesidad de cultivar la teoría por encima de otras consideraciones: enfocar la ciencia en vista de sólo las aplicaciones se le muestra imposible porque si se suprime la teoría cualquier tipo de progreso se detendría. Además, la ciencia es uno de los logros más altos del espíritu humano, en sus palabras, con un lejano tinte del Macbeth shakespeareano:

Sólo por la Ciencia y por el Arte valen las civilizaciones. Se ha sorprendido de esta fórmula: la Ciencia por la Ciencia; $y$ sin embargo, ello vale tanto como la vida por la vida, aunque la vida no sea más que miseria. (...).

Debemos sufrir, debemos trabajar, debemos pagar nuestro lugar en el espectáculo, pero es para ver; o al menos para que otros vean un día. (VC $\mathrm{p}$. 275).

Desde esta posición, estar al servicio de la sociedad implica trabajar para que otros vean, no sólo quien trabaja. Y es un papel que también ejerce Poincaré en varios frentes, en al menos los cuatro siguientes:

\section{Profesional}

Es el terreno en el que todos nos movemos. Cada uno ha de pertenecer a un ámbito profesional determinado y, desde él, ejercer esa función de matemático con sus diferentes papeles. Pero también, en esta profesión, además de ejercer la docencia, ha de emitir informes, dirigir tesis, pertenecer a múltiples comisiones... Poincaré fue el único miembro electo de todas las secciones de la Academia de Ciencias francesa del momento -geometría, mecánica, física, geografía, navegación- y en 1906 fue elegido Presidente de la misma; en 1908 elegido de la Academia de la Lengua de la cual, en 1912, se convirtió en su Presidente. Como académico, emitió informes de todo tipo, hasta le correspondió el estudio de la nueva medición de la longitud del Ecuador llevada a cabo desde Quito, potenciar el hallazgo de los rayos X por parte de Becquerel, ayudar a Marie Curie en sus investigaciones, recomendar a Einstein para profesor en Zürich... También como profesional cabe recibir algún premio, alguna distinción: Poincaré recibió multitud de honores, premios y distinciones. 
Experto

La sociedad, en un momento determinado, puede encontrarse dividida en algunas cuestiones y, para tratar de dirimirlas, acude al experto, a quien considera capaz de orientar la posible solución. El affaire Dreyfus provocó una auténtica escisión en la sociedad francesa a finales del s. XIX. Se dirimían, en el fondo, cuestiones de prestigio consecuencia de la guerra franco prusiana con la derrota de Sedán, la pérdida de la Lorena; la cuestión judía; los problemas religiosos y si el Estado debería ser laico o mantener una determinada confesionalidad... No sólo Zola sale a polémica pública sino algunos matemáticos, especialmente normalianos, publican el «manifiesto de los matemáticos» encabezado por Hadamard, por Painlevé. En 1904 -y estamos en el centenario, uno más- la Corte Suprema de Francia designa, para intentar solventar este affaire, una Comisión de Expertos: Appell, Darboux, Poincaré. Se trata de decidir si el escrito acusatorio es, como quieren los especialistas grafólogos, de la mano de Dreyfus. Es una de las únicas pruebas en las que se apoyaba la acusación y por la cual se había condenado al militar judío-francés Dreyfus por espía al servicio alemán. Appel, Darboux, Poincaré tienen que decidir, como expertos - no sé si en grafología- el tema. Como contará Appel posteriormente es Poincaré quien hace el estudio y redacta el informe que hace suyo la Comisión de expertos.

Poincaré recaba la ayuda del Observatorio de París y maneja la última invención tecnológica, el micrómetro, con el cual se dedica a medir el intervalo entre cada letra, su altura, la inclinación que tienen entre sí y con la hoja total... Mediciones que se encuentran entre los papeles de Poincaré en los Archivos de la Universidad de Nancy. Mediciones con precisión de milésimas de milímetro. Y tras esas mediciones, un informe durísimo y que constituirá la apoyatura para que la Corte Suprema decida la definitiva resolución del caso con la libre absolución y restitución para Dreyfus.

Los expertos han cumplido su papel. No sólo eso: Poincaré, en el informe, va a señalar, de modo radical, que el tema no es científico sino ideológico y político y, por ello, no se debe mezclar con lo científico. Los peritos grafólogos habían intentado, en su estudio, ayudarse del Cálculo de Probabilidades y Poincaré va a rechazar, radicalmente, ese uso. Se le muestra como un uso ilícito de la ciencia. Para dirimir cuestiones ideológicas, políticas, religiosas no se debe acudir a la ciencia sino a la ideología, la política, la religión. 
Crítico social

El papel de experto al que se ve llevado en el caso anterior conduce a radicalizar el pensamiento de Poincaré en el sentido de que también el científico debe entrar a delimitar su papel en la sociedad, debe establecer cuál es su función en ella. Y aquí Poincaré entra en el terreno de la Moral -donde los principios son normativos, y donde lo que impera es el debe-distinguiéndolo del terreno de la Ciencia -donde los principios son declarativos, y en los que impera el es-. Una diferencia que le conduce a mantener la distinción entre la Moral y la Ciencia. De otra manera se cometería la clásica falacia de identificar es con debe.

Es un tema de preocupación constante en Poincaré. Como científico niega que la ciencia pueda fundamentar la moral e insistirá en su rechazo al mal uso de la ciencia, a un uso interesado y tergiversado. No se puede utilizar la ciencia al servicio de unas ideologías como insistirá en su debate con Le Roy, en el fondo con Duhem, quienes manejan la ciencia y su historia en defensa de la religión católica, por ejemplo, y hacen lo que alguno llegó a calificar entonces de "ciencia del creyente».

Terreno, el de la moral, en el que plantea cuestiones que siguen siendo de actualidad como el masivo empleo de la estadística en la vida ordinaria, la problemática de la vivisección, de las prácticas médicas y biológicas con animales, práctica que defiende de modo radical. Tampoco deja a un lado temas como el de la educación que se le muestra como esencial para el progreso moral de los individuos.

Es campo en el que defenderá, a ultranza, la libertad de la Ciencia, del pensamiento científico y su separación de cualquier tipo de dogma, religión o partido. En 1909, en su alocución Le libre examen en matiére scientifique, afirma

El pensamiento no debe someterse nunca ni a un dogma, ni a un partido, ni a una pasión, ni a un interés, ni a una idea preconcebida, ni a nada, si no es a los hechos mismos, porque, para él, someterse sería dejar de ser. (1909, p. 139).

La Ciencia, la Matemática, es un producto de la razón humana, lo más digno de ella y frente a quienes sostienen la existencia de lo inefable, captable sólo por algo distinto a esa razón, alzará su voz

Todo lo que no es pensado es la pura nada, ya que no podemos pensar más que el pensamiento y todas las palabras de las que disponemos para hablar de las cosas no pueden expresar más que pensamientos; decir que hay otra cosa que el pensamiento, es pues una afirmación que no puede tener sentido. (VC p. 276). 
Divulgador científico

El matemático, el científico, vive en sociedad. Y los miembros de esa sociedad deben conocer lo que se hace en la ciencia. Surge, así, otra función para el matemático: divulgar su saber. Una divulgación que le corresponde hacer al científico creador, no al simple periodista. Sólo quien investiga en un sector sabe los temas, las dificultades, las líneas posibles, los fracasos que se ligan a esa investigación. Por ello es él quien debe hacer el esfuerzo y poner al alcance del gran público los grandes temas y problemas de la ciencia en cada momento.

Cumpliendo esta labor Poincaré reune alguno de sus escritos en libros que se convierten en auténticos best-seller en una época en la cual la considerada divulgación científica era prácticamente inexistente o se centraba en obras como las de Julio Verne.

En Diciembre de 1902 sale a luz Ciencia e Hipótesis; en 1905, El valor de la Ciencia; en 1908, Ciencia y Método. Un año después de morir, en 1913, se edita Últimos pensamientos. Son los libros que cimentan su popularidad y no sólo entre el gran público. Popularidad unida a su aureola de sabio, de cerebro viviente de las ciencias racionales como lo calificara Painlevé. Auténticas obras de difusión, las cifras de ejemplares en 1914 llegan a más de 21.000 para Ciencia e Hipótesis y para El valor de la ciencia; 12.100 para Ciencia y Método; 7.700 para Últimos pensamientos. Un total aproximado de 61.700 ejemplares vendidos entre Diciembre de 1902 y 1913. A ellos habría que agregar los correspondientes a las traducciones al alemán, inglés, español, sueco, húngaro, japonés realizadas de Ciencia e Hipótesis antes de $1910 .$.

Publicaciones estimadas de alta divulgación, encierran más de un problema. Para hacer su pensamiento accesible al gran público Poincaré suprime las referencias matemáticas, elimina fórmulas, ecuaciones, gran parte del razonamiento estrictamente matemático que, sin embargo, permanecen subyacentes; une o trocea distintos ensayos. Son eliminaciones que, en ocasiones, llevan a confusiones al lector no atento. La claridad y aparente simplicidad de estos ensayos, su espléndida limpidez, oculta un pensamiento profundo. Luis Rougier, en 1919, cuando pretendía la publicación de un quinto volumen de ensayos de Poincaré escribía a León Daum, representante de la familia del matemático francés, que de entre los 24.000 lectores de Ciencia e Hipótesis probablemente sólo unos mil habrían comprendido el libro. Y, ciertamente, ha habido y hay muy distintas interpretaciones de ese pensamiento tan aparentemente simple, a veces, de Poincaré. 
En cualquier caso constituye una de las caras a las que el matemático debe someterse: la de divulgador, con todas las dificultades que ella conlleva. Pero Poincaré no sólo hace ese papel con los cuatro libros mencionados. También dedica su tiempo a divulgar temas de electricidad, de telegrafía en las revistas que las asociaciones de Electricistas, de Telégrafos editan. Una labor que completa la anterior.

\section{Una respuesta insospechada: Los 23 problemas de hilbert}

He intentado exponer, brevemente, lo que puede calificarse Programa Poincaré para la Matemática del futuro, programa centrado en las funciones que asigna al matemático, tanto las explícitas como las que, de manera intrínseca, ejemplifica con su trabajo. Un Programa que va a tener una repercusión un tanto inesperada e insospechada para la mayoría. En 1900 se celebra en París el $2^{\circ}$ CIM y los organizadores invitan a participar en él a David Hilbert. Hemos conmemorado, en el 2000, el Año Mundial de las Matemáticas en recuerdo, precisamente, del programa hilbertiano. Si en 1900 Hilbert enuncia 23 problemas futuros, los problemas que estima básicos para la Matemática del s. XX, en el año 2000 se ha pretendido una retrospectiva de lo adquirido e indicar, también, las líneas futuras, los problemas centrales para el primer siglo de este nuevo milenio.

Cuando Hilbert recibe la invitación para el CIM decide que su intervención sea una respuesta explícita a las palabras, al Programa Poincaré, y así lo comunica a sus dos fieles amigos y asesores, Hurwitz y Minkowski. Este, en carta de 5 de Enero de 1900, contesta aconsejando no responder de modo directo porque supondría entrar en terrenos de naturaleza filosófica «que son probablemente mejores para una audiencia alemana» que no francesa y en cambio sugiere

Más atractivo sería el intento de mirar al futuro, en otras palabras, de hacer una caracterización de los problemas a los que los matemáticos deberían orientarse en el futuro. Con esto podrías tener a la gente hablando de tu charla incluso durante décadas a partir de ahora. Por supuesto, la profecía es realmente asunto difícil. (De Gray, p. 70).

Algo que tendría el valor añadido de hacerlo, precisamente, en París, ante los matemáticos franceses. Hilbert hace caso del consejo de Minkowski como hemos visto y vivido porque la profecía de Minkowski se ha cumplido, y con creces. Pero no por ello Hilbert deja de responder, ahora 
de manera implícita, a Poincaré lo que se observa en, al menos, tres aspectos:

- en la autolimitación a la «matemática pura», planteando problemas futuros internos a la misma, de los que se afirma que pueden ser definitivamente resueltos; y cuando plantea un problema de Física, el problema se centra en exigir que la Física se axiomatice, se formalice;

- en la afirmación de que si en un origen remoto la Matemática surgió de la experiencia, de la necesidad de responder a la misma, posteriormente ha sido y es la propia razón matemática la que origina los problemas sin intervención extraña, sin dependencia o interrelaciones con otras disciplinas; todo lo opuesto a estimar como válidas las interrelaciones entre Física y Matemática, todo lo contrario a la afirmación de Poincaré de que el espíritu de ambas es el mismo;

- en la elección de los problemas planteados como claves para el desarrollo futuro de la Matemática. En este punto no hay que fijarse tanto en la elección explícita de los problemas -todos ligados, realmente, a la escuela alemana, fundamentalmente a la que se está creando en Göttingen-, como en aquellos que deja a un lado y que van a ser, sin embargo, fundamentales para el Hacer matemático del s. XX: son problemas en los cuales trabajan básicamente los matemáticos franceses -y que dan paso a la Teoría de la medida con la integral-Lebesgue o el análisis funcional...-y, en particular, las cuestiones ligadas a la Topología, a la geometrización de la matemática, al problema de los tres cuerpos en dinámica..., a los temas en los que trabaja Poincaré. Lo mismo que deja a un lado la Teoría de la probabilidad, la teoría de matrices, la lógica formal iniciada por Frege y Peano... temas a los cuales, y como auténtico contraste, dedicará Hilbert su atención en los años siguientes como, por ejemplo, al análisis funcional en relación con las ecuaciones integrales. Contraste con las líneas que él mismo señala para el futuro de la Matemática y en las cuales parece no creer tan radicalmente...

Al dar cuenta del Congreso en carta a Hurwitz a finales de Agosto, Hilbert no menciona su propia intervención pero señala dos puntos a destacar: su desilusión por la poca calidad de las comunicaciones y, sobre todo, el hecho de que Poincaré estuvo presente en el CIM única y exclusivamente por necesaria obligación...

Que Hilbert concibió su lectura como réplica al Programa Poincaré de 1897 es algo intuido, sin conocer quizá la correspondencia, por Grattan- 
Guinnes quien menciona el «(aparente) silencio» de Poincaré ante esta comunicación. Silencio que interpreta como una respuesta del tipo

Intuición y aplicaciones por favor, querido colega, no todo es axiomática purista. (p. 757).

El Programa Poincaré tuvo, así, unas consecuencias para el hacer matemático del s. XX un tanto insospechadas al obligar a plantear a los matemáticos alemanes, en el fondo, un programa alternativo para el Hacer matemático del s. XX, especialmente a la escuela naciente de Gottingen...

Alternativo porque Poincaré, en el Cuarto CIM celebrado en Roma en 1908, reitera el suyo, insiste en las funciones del matemático o, en otras palabras, en que la Matemática confina con la Física y la Filosofía. Ahora, y de modo ya explícito, titula su Ponencia El porvenir de las $\mathrm{Ma}$ temáticas, donde también hay respuesta, por supuesto que implícita, a los problemas inventariados por Hilbert. Aquí no se plantean unos determinados problemas porque ello supondría realizar un inventario de un mundo ya cerrado, ya acabado y he indicado que, para Poincaré, la Matemática es un hacer siempre abierto, producto de la razón humana y donde

Los problemas insolubles se han convertido en los más interesantes de todos, porque han planteado problemas en que ni siquiera se había soñado. (CM p. 23)

y, por ello, no estaban previamente inventariados ni se los puede inventariar. Por otro lado, se puede afirmar que, en cierto sentido, los problemas más interesantes son aquellos de los que se puede decir que no han sido definitivamente resueltos sino que están más o menos resueltos

No hay problemas resueltos y otros que no lo están, sólo hay problemas más o menos resueltos (id.)

Parece tener en mente las ecuaciones diferenciales cualitativas...

Por supuesto, la insistencia en el papel de la geometría como elemento clave tanto en sí como en su papel heurístico: cualquier disciplina matemática viene subtendida por la intuición geométrica; perderla constituiría un error que es lo que se produce en el formalismo hilbertiano, en los fundamentalismos cantorianos o conjuntistas, en las pretensiones de los logicistas.

Dos Programas en los que está en juego la existencia de dos conceptos del Hacer matemático: un hacer que se limita a una matemática 
"pura» con problemas estrictamente internos y uno que considera que no puede disociarse de otros haceres porque encerrarse en una torre de marfil terminará en una formalización sintáctica prácticamente estéril. Consecuente, distintos enfoques en cuanto a la necesidad de los formalismos, de la derivación o de la demostración intuitiva matemática...

Habría que precisar que el programa finitista constructivo que plantea Poincaré, con la necesidad de la estructura formal en la cual la noción de grupo se le presenta básica, va a ser recogido por Hilbert desde los años veinte en su programa estrictamente finitista pero con un giro esencial: su apoyatura en lo puramente sintáctico formal, con las conse cuencias que todos conocemos, especialmente tras el papel atribuido al bourbakismo.

Aunque también es de justicia reconocer que Hilbert se ocupará de la Física teórica a partir de 1904 -y en contra, una vez más, de su inventario, del credo que explicita en su alocución de 1900- en ese proceso de tratar de liderar la Escuela de Göttingen y potenciar, en ella, y a través de diferentes colaboradores y con trabajo no ya individual sino de equipo, todos los aspectos del Hacer matemático así como sus enlaces con otras disciplinas.

No deseo terminar sin citar un largo párrafo de V. I. Arnold escrito con motivo de las actividades del Año Mundial de las Matemáticas e inspirado en la lista o inventario de problemas propuestos por Hilbert en 1900. Arnold ha captado, aunque de modo implícito, la existencia de los dos programas y lo que hace, realmente, es una comparación entre las influencias que ambos han tenido en la matemática del s. XX. Las palabras con las que finaliza su ensayo, un tanto parciales y en cualquier caso con cierto aire polémico, son:

Hilbert intenta predecir el desarrollo futuro de las matemáticas e influir en él por sus Problemas. El desarrollo de la matemática en el siglo 20 ha seguido un camino diferente. Los logros más importantes -el florecimiento de la teoría de la homotopía y la topología diferencial, la geometrización de todas las ramas de la matemática, su fusión con la física teórica, el descubrimiento de problemas algoritmicamente indecidibles y la aparición de los ordenadores- todo esto ha ido en dirección diferente (si no opuesta).

La influencia de H. Poincaré y de H. Weyl sobre la ciencia del s. 20 ha sido más profunda. Para Poincaré, quien creó la matemática moderna, topología y teoría de sistemas dinámicos, el futuro de la matemática se enlaza con el desarrollo de la física matemática, orientado a la descripción de la teoría relativista y cuántica. Entre otras cosas importantes, Poincaré explicaba que sólo los problemas no-interesantes pueden ser formulados sin ambigüedad y resueltos completamente. Según Poincaré uno debería intentar comprender lo 


\section{El «Programa Poincaré» o funciones del matemático}

que debe ser cambiado en la formulación del problema. Ante todo tenía en el espiritu la variación de los coeficientes de las ecuaciones problema del tipo bifurcación y todos los tipos de argumentos de posición general -los tópicos que ahora se llaman teoría de la singularidad, análisis global y análisis funcional. Bastante interesante, lo que ahora se llama teorema de la deformación versal ha sido demostrado en su Tesis (para el caso de las intersecciones holomorfas completas de dimensión cero) como lema 4, y fue la base para su teoría de la bifurcación. (Arnold 2000, pp. 415-416)

\section{Referencias}

Las citas de Poincaré se encuentran en:

[CH] Poincaré, H. (2002, primera edición de 1902) Ciencia e Hipótesis. Ed: Espasa Calpe, Madrid. Introducción de Javier de Lorenzo

[VC] Poincaré, H. (1948, primera edición de 1905) La valeur de la Science. Ed: Flammarion, París.

[CM] Poincaré, H. (1963, primera edición de 1908) Ciencia y Método. Ed: Espasa Calpe, Madrid. Traducción de García Miranda y L. Alonso.

Los datos de difusión de las obras de Poincaré han sido tomados del prefacio de

[1] Rollet, L. (2002) Henri Poincaré: Scientific Opportunism. An Anthology. Ed: Birkhauser.

Para el tema de los expertos:

[2] Rollet, L. (1997) Autour de l'affaire Dreyfus. Revue Historique 603 (Julio-Septiembre), 49-101.

Las demás citas o menciones se encuentran en:

[3] Arnold, V.I. (2000) Polymathematics: Is Mathematics a Single Science or a Set of Arts? En: Mathematics: Frontiers and Perspectives 2000. Editores: Arnold, Atiyah, Lax y Mazur. Ed: International Mathematical Union. AMS. Páginas $403-416$.

[4] Grattan-Guinness, I. (2000) A Sideways Look at Hilbert's Twenty-three Problems of 1900. Notices of the AMS 47(7) 752-757.

[5] Gray, J.J. (2003) El reto de Hilbert. Ed: Crítica, Barcelona. Traducción de J. García Sanz.

[6] Lorenzo, J. de (1974) La filosofía de la matemática de Poincaré. Ed: Tecnos, Madrid.

[7] Lorenzo, J. de (publicación probable en 2004) Poincaré, pensador de la matemática. En: Conferències de la FME: Curs Poincaré 2003-2004. Ed: Universidad Politécnica de Barcelona. 